\title{
Two cases of TSC2/PKD1 contiguous gene deletion syndrome
}

\author{
Jihye You', Eungu Kang', Yoonmyung Kim¹, Beom Hee Lee', Tae-Sung Ko ${ }^{1,2}$, Gu-Hwan Kim², Jin-Ho Choi', and Han-Wook Yoo ${ }^{1,2, *}$ \\ 'Department of Pediatrics, Asan Medical Center Children's Hospital, University of Ulsan College of Medicine, Seoul, Korea \\ ${ }^{2}$ Medical Genetics Center, Asan Medical Center, University of Ulsan College of Medicine, Seoul, Korea
}

Tuberous sclerosis complex (TSC, MIM\#191100) is an autosomal dominant neurocutaneous syndrome caused by mutation or deletion of TSC1 encoding hamartin or TSC2 encoding tuberin and characterized by seizure, mental retardation, and multiple hamartomas or benign tumors in the skin, brain, retina, heart, kidney, and lungs. The TSC2 gene on chromosome 16 13.3 lies adjacent to the $P K D 1$ gene which is responsible for autosomal dominant polycystic kidney disease (MIM\#173900). The TSC2/PKD1 contiguous gene syndrome (TSC2/PKD1 CGDS, MIM\#600273) is caused by deletion of both TSC2 and PKD1 gene. We recently experienced a 15 month-old boy and a 26 month-old girl with TSC2/PKD1 CGDS confirmed by multiplex ligation-dependent probe amplification (MLPA) analysis. They showed not only typical neurologic manifestations of TSC such as epilepsy, subependymal nodules, and subcortical tubers, but also polycystic kidney disease. The contiguous gene syndrome involving PKD1 and TSC2 should be suspected in children with enlarged polycystic kidneys and TSC. MLPA analysis is a useful method for the genetic confirmation of TSC2/PKD1 CGDS.

Key words: Tuberous sclerosis, Autosomal dominant polycystic kidney disease, Polycystic kidneys, severe infantile with tuberous sclerosis, Multiplex polymerase chain reaction.

\section{Introduction}

Tuberous sclerosis complex (TSC, MIM\# 191100) is an autosomal dominant syndrome characterized by seizure, mental retardation, and multiple hamartomas in the brain, heart, skin, retina, kidneys, or liver. Random distributions of tumorous lesions cause variable features such as neurologic deficits, renal impairment, skin lesions, or retinal hamartomas. The gene mutation can be identified in $85 \%$ of patients: TSC1 on chromosome $9 q 34$, encoding hamartin, or TSC2 on 16p13.3, encoding tuberin [1]. The incidence of TSC is estimated to be 1 in 6,000-11,000 births
[2]. Sporadic cases constitute two thirds of tuberous sclerosis cases. TSC2 mutations are about four times more frequent in sporadic cases compared to TSC1 mutations and cause a more severe phenotype [3].

Autosomal dominant polycystic kidney disease (ADPKD, MIM\# 173900) is a common renal disorder, occurring in approximately 1 in every 1,000 live births [4]. It is characterized by progressive bilateral renal cysts leading to renal failure in the fifth to seventh decade of life. Occasionally, liver cysts and intracranial aneurysm may occur. Mutations in PKD1 on 16p13.3, encoding polycystin 1, or PKD2 on 4q22, encoding

Received: 28 April 2016, Revised: 30 May 2016, Accepted: 31 May 2016, Published: 30 June 2016

*Corresponding author: Han-Wook Yoo, M.D., Ph.D.

Department of Pediatrics, Asan Medical Center Children's Hospital, University of Ulsan College of Medicine, 88 Olympic-ro 43-gil, Songpa-gu, Seoul 05505, Korea.

Tel: +82-2-3010-3374, Fax: +82-2-473-3725, E-mail: hwyoo@amc.seoul.kr

Conflict of interest: The authors declare that they do not have any conflicts of interest.

(c) This is an open-access article distributed under the terms of the Creative Commons Attribution Non-Commercial License (http://creativecommons.org/licenses/by-nc/4.0/) which permits unrestricted non-commercial use, distribution, and reproduction in any medium, provided the original work is properly cited.

(c) Copyright 2016 by the Korean Society of Medical Genetics 
polycystin 2, have been confirmed to cause ADPKD. PKD1 gene mutations have been reported in 85\% of patients, and 2-3\% of these mutations are caused by large deletions [5].

TSC2/PKD1 contiguous gene deletion syndrome (TSC2/PKD1 CGDS, MIM\#600273) was first recognized by Brook-Carter et al. [6] in 1994 in a patient with tuberous sclerosis and cystic renal disease by identifying the deletion of both TSC2 and PKD1 genes. TSC2/PKD1 CGDS can occur due to the adjacent location of the TSC2 and PKD1 genes. More cases have been reported in TSC patients with enlarged polycystic kidneys [7-14]. Although 5\% of patients with TSC have TSC2/PKD1 CGDS, there have been no report of patients with TSC2/PKD1 CGDS in the Korean population. This study describes two cases of tuberous sclerosis with severe cystic renal disease, which were diagnosed with TSC2/PKD1 CGDS by multiplex ligation-dependent probe amplification (MLPA) analysis.

\section{Case}

\section{Case 1}

The patient is the first baby of non-consanguineous Korean parents. He was born after 39 weeks of gestation with a birth weight of $3.12 \mathrm{~kg}$, without any perinatal problems. At 5 months of age, he was brought to the outpatient clinic due to heart murmur and multiple cardiac masses. Two cardiac masses were located in the right ventricular outflow tract and right ventricular free wall at the anterior leaflet of the tricuspid valve (Fig. 1A). In addition, bilateral multiple renal cysts were found on the renal ultrasound (Fig. 1B).
At age 12 months, generalized tonic-clonic seizure developed, which was provoked by fever. The seizure persisted over 15 minutes with cyanosis, followed by postictal sleep. It was clustered, with 5 seizures occurring in one episode. His blood pressure was 103/45 mm $\mathrm{mm}$, and his pulse rate was 108 beats per minute. His height and weight were $85.4 \mathrm{~cm}$ (standard deviation [SD], 0.87) and $12.7 \mathrm{~kg}(\mathrm{SD}, 0.73)$, respectively. His head circumference was $48 \mathrm{~cm}(S D, 0.17)$. He had a hypopigmented nevus on the nape, but did not have palpable abdominal mass. Complete blood counts, serum electrolytes, liver enzymes, and routine urinalysis were all normal. Brain magnetic resonance imaging (MRI) revealed multiple subependymal nodules in the foramen of Monro and multiple cortical tubers with subcortical white matter changes in both cerebral hemispheres (Fig. 1C). Multiple retinal tumors were found upon ophthalmologic examination. There was no familial history of tuberous sclerosis and ADPKD. Phenobarbital was administered to control the seizures, but was discontinued after 14 days because of adverse events such as skin rash and pruritus. He did not experience an additional seizure until age 30 months, when his height and weight were $95.6 \mathrm{~cm}(S D, 1.54)$ and $14.8 \mathrm{~kg}$ (SD, 0.85), respectively. His development was also normal.

For genetic testing, informed consent was obtained from both parents. Genomic DNA was isolated from the patient's peripheral leukocytes. MLPA analysis using probe-mixes for TSC2 and PKD1, P046-B2 and P351-B2 (MRC-Holland, Amsterdam, The Netherlands), identified a large deletion of $P K D 1$ from exons 39 to 46 and TSC2 from exons 26 to 41 (Fig. 2A).

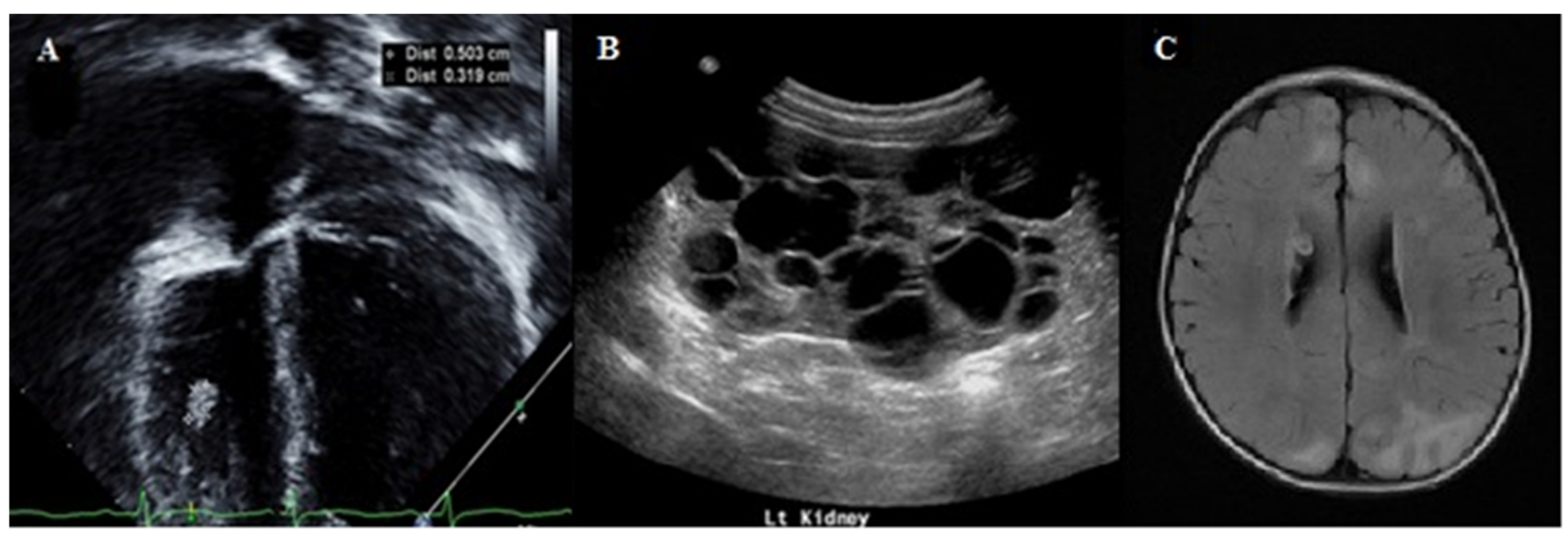

Fig. 1. Cardiac, renal and neurological findings of patient 1. (A) Echocardiography shows two cardiac masses on the right ventricular free wall. (B) Bilateral enlarged kidneys with multiple cysts are noted in renal ultrasonography (left kidney length, $11.8 \mathrm{~cm}$ ). (C) Brain magnetic resonance image reveals multiple cortical tubers with high signal intensities in subcortical white matter and radial white matter bands in both cerebral hemispheres and multiple subependymal nodules with or without calcifications in axial T2 fluid-attenuated inversion recovery (FLAIR) image. 


\section{A MLPA analysis of TSC2 and PKD1 gene. (P046-B2; P351-B2, MRC Holland)}

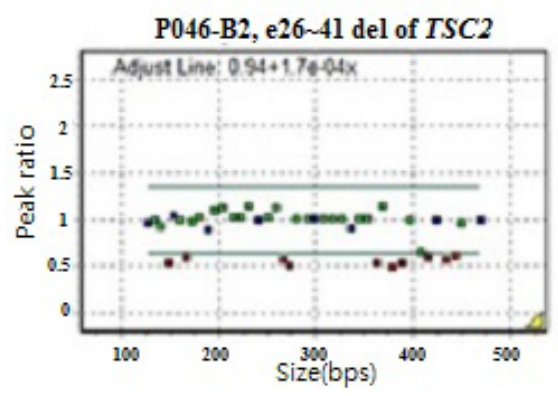

B

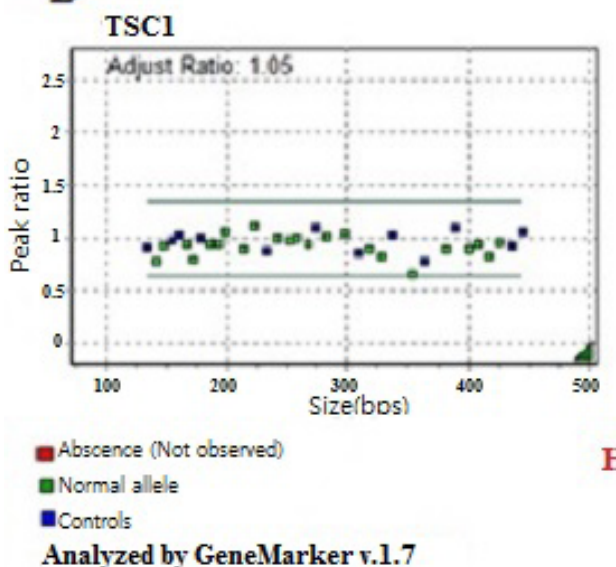

P351-B2, e39 46 del of PKD1

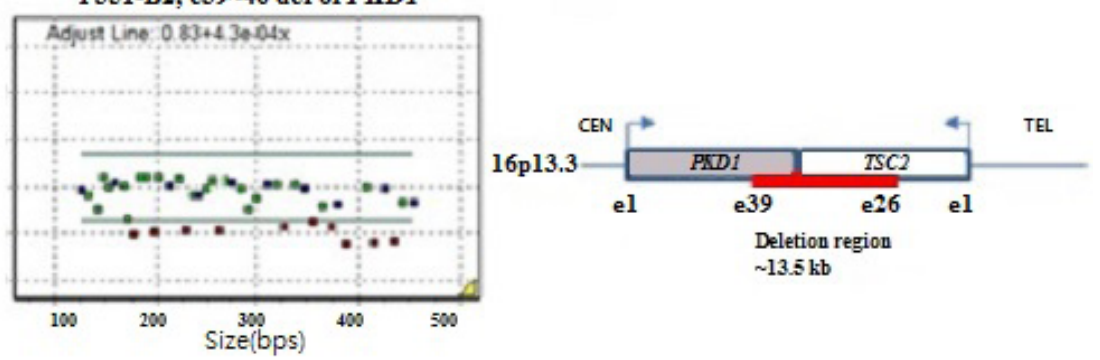

TSC2

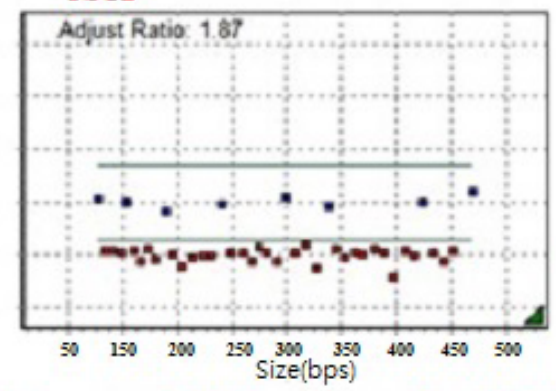

Heterozygous deletion at exon 1 to 41 of $T S C 2$ and exon 46 of $P K D 1$

Fig. 2. The genetic findings of patient 1 and 2. (A) Multiplex ligation-dependent probe amplification (MLPA) analysis of Case 1 shows large deletion of PKD1 from exon 39 to exon 46 and contiguous TSC2 deletion from exon 26 to 41 by probe-mixes for TSC2 and PKD1, P046-B2 and P351-B2 (MRC-Holland, Amsterdam, The Netherlands). (B) Whole exonic deletions of TSC2 and PKD1 are showed in MLPA analysis in Case 2.

\section{Case 2}

The patient was born prematurely after 35 weeks of gestation with a birth weight of $2.3 \mathrm{~kg}$ due to preterm premature rupture of membrane. At 2 months of age, $\beta$-blocker was administered because of left forearm hemangioma. At 5 months of age, an atrial septal defect was observed by echocardiography, without a cardiac mass. Hypopigmented macules were detected on the left lower leg and anterior chest. At 8 months of age, generalized tonic-clonic seizure developed with fever, and redeveloped at age 10 months. At 26 months of age, she was referred to the emergency room because of recurrent seizures with cyanosis and loss of consciousness, which repeated 6 times within a day. On physical examination, blood pressure was $98 / 56 \mathrm{mmHg}$ and pulse rate was 132 beats per minute. Height and weight were $90.0 \mathrm{~cm}(S D, 0.45)$ and $14.4 \mathrm{~kg}(S D, 1.74)$, respectively. Facial dysmorphism or adenoma sebaceum was not found. She had hemangioma on the left forearm and hypopigmented patches on the anterior chest, left shoulder, and left leg. There was no palpable abdominal mass. Complete blood counts, liver enzymes, and other blood chemical tests were normal. Brain MRI revealed multiple cortical and subcortical tubers in bilateral hemispheres and several tiny subependymal hamartomas along the wall of both lateral ventricles (Fig. 3A). On the electroencephalogram, occasional theta or delta slow wave discharges from left frontotemporo-parietal areas were detected (Fig. 3B). No anatomical anomaly was detected by echocardiography. Abdominal sonogram demonstrated small echogenic oval lesions without increased vascularity, suggesting angiomyolipoma in the left kidney middle pole, and multiple small $(<1 \mathrm{~cm})$ cysts in both kidneys (Fig. 3C). Ophthalmologic examination detected yellowish elevated lesions near the superior temporal vascular arcade on the right fundus, suggesting retinal hamartoma. There was no familial history of tuberous sclerosis and ADPKD.

MLPA analysis identified heterozygous deletion at all 41 exons of TSC2 and at exon 46 of PKD 1 (Fig. 2B). Since whole PKD1 MLPA was not conducted, the exact extent of exon deletion was not identified. However, we can guess that the deletion may disrupt parts of the PKD 1 gene, as the deletion of exon 46 in 

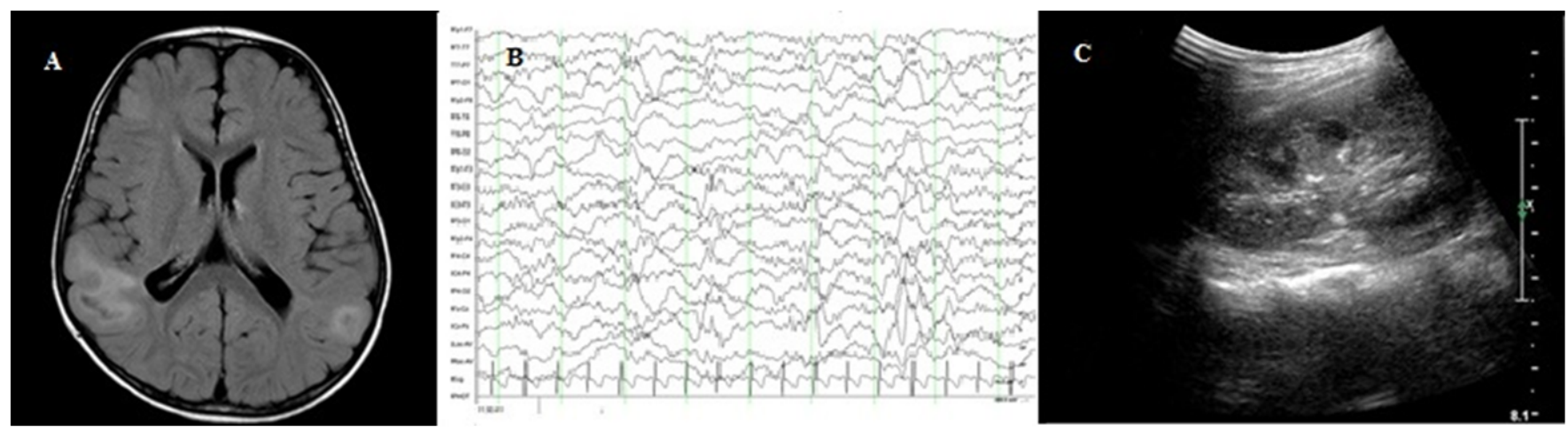

Fig. 3. Neurological and renal findings of patient 2. (A) Brain magnetic resonance image demonstrates multiple cortical and subcortical tubers with high signal intensities expanding gyri in bilateral hemisphere and multiple small subependymal nodules along wall of both ventricles without ventriculomegaly in axial T2 fluid-attenuated inversion recovery (FLAIR) image. (B) 0ccasional theta or delta slow wave discharges from left fronto-temporo-parietal areas were detected on electroencephalogram. (C) Kidney ultrasonography shows multiple small cysts in both kidneys. A small angiomyolipoma is seen in the middle pole of left kidney (left kidney length, $6.1 \mathrm{~cm}$ ).

PKD1 has been identified.

\section{Discussion}

In the current report, we described two cases of gross deletions in the TSC2 and PKD1 genes in Korean patients with TSC and ADPKD. This is the first report of TSC2/PKD1 CGDS in the Korean population. The large deletion involved exons 39 to 46 of PKD 1 and exons 26 to 41 of TSC2, as revealed in Case 1 by MLPA. Although the precise length of deletion of the PKD1 gene was not identified, Case 2 also showed the large deletion involved TSC2 and PKD1. As the TSC2 gene and the PKD 1 gene lie adjacent to each other in a tail-to-tail arrangement, it is possible that deletion of TSC2 can occur together with PKD1 deletion, leading to contiguous gene deletion syndrome.

Neurologic manifestation is the most common cause of morbidity and mortality in patients with tuberous sclerosis, followed by renal involvement. Renal angiomyolipoma is a common feature of tuberous sclerosis and leads to hemorrhage or torsion. In comparison to the renal manifestation of tuberous sclerosis, renal cystic disease is more common in ADPKD, causing hypertension, proteinuria and end-stage renal disease. In TSC2/ PKD1 CGDS, severe, infantile or childhood onset polycystic kidney disease is more frequent than isolated TSC or ADPKD [15]. The cysts are larger and space-occupying, subsequently causing hypertension and early onset end-stage kidney disease. More importantly, the regular radiological follow-up should be recommended because of the risk of malignant transformation [7]. Five percent of patients with tuberous sclerosis and nearly $10 \%$ of patients with ADPKD were reported to have renal cell carcinoma at a young age [16]. Although the frequency of renal cell carcinoma in TSC2/PKD1 CGDS is unknown, the risk of renal cell carcinoma is expected to be high.

The mechanism for renal cyst formation remains elusive, though hamartin, tuberin, and polycystin-1 have been known to play a role in maintaining cellular polarity and the length of primary cilia in pre-cystic renal tubule cells [17]. The mutations in TSC2 and PKD 1 seem to produce a truncated tuberin which activates guanosine triphosphatase (GTPase), leading to a reduction in mammalian target of rapamycin (mTOR) stimulation. The disruption of these genes was expected to result in cyst enlargement through mTOR activation [9]. In recent studies, however, mTOR inhibitors were not successful in treating the renal involvement of TSC and ADPKD. Instead, mTOR inhibitor was effective in reducing angiomyolipoma volume in patients with TSC [18]. In patients with TSC2/PKD1 CGDS as well, mTOR inhibitor was not effective for improving renal function, but produced the satisfactory effect of renal angiomyolipoma size reduction [9]. Renal involvement was shown in our patients even at the age of 12 months.

The extrarenal manifestations of TSC2/PKD 1 CGDS are not clearly identified. One report suggests that the pathological features of skin lesions from patients with contiguous gene syndrome were similar to those from patients with tuberous sclerosis [13]. It remains to be seen whether the neurologic, ocular, or cardiac manifestations in patients with this contiguous gene syndrome differ from those in patients with isolated tuberous sclerosis. A few case reports described the extrarenal manifestations of ADPKD, such as intracranial aneurysm with clinical manifestations of TSC [19]. Therefore, TSC2/PKD1 CGDS should be suspected in those with extrarenal symptoms of ADPKD, such as visceral organ cysts, or intracranial aneurysms 
additional to the clinical signs of TSC.

In conclusion, the contiguous gene syndrome involving PKD1 and TSC2 should be considered in patients with ADPKD and TSC. MLPA analysis has a confirmatory role in the diagnosis of TSC2/ PKD1 CGDS.

\section{Acknowledgements}

This study was supported by a grant from the National Research Foundation of Korea, funded by the Ministry of Education, Science, and Technology (2011-0019674).

\section{References}

1. European Chromosome 16 Tuberous Sclerosis Consortium. Identification and characterization of the tuberous sclerosis gene on chromosome 16 . Cell 1993;75:1305-15.

2. Osborne JP, Fryer A, Webb D. Epidemiology of tuberous sclerosis. Ann N Y Acad Sci 1991;615:125-7.

3. Dabora SL, Jozwiak S, Franz DN, Roberts PS, Nieto A, Chung J, et al. Mutational analysis in a cohort of 224 tuberous sclerosis patients indicates increased severity of TSC2, compared with TSC1, disease in multiple organs. Am J Hum Genet 2001;68:64-80.

4. Gabow PA. Autosomal dominant polycystic kidney disease. N Engl J Med 1993;329:332-42.

5. Fick GM, Gabow PA. Hereditary and acquired cystic disease of the kidney. Kidney Int 1994;46:951-64.

6. Brook-Carter PT, Peral B, Ward CJ, Thompson P, Hughes J, Maheshwar MM, et al. Deletion of the TSC2 and PKD1 genes associated with severe infantile polycystic kidney disease--a contiguous gene syndrome. Nat Genet 1994;8:328-32.

7. Paul $E$, Thiele EA, Shailam R, Rosales AM, Sadow PM. Case records of the Massachusetts general hospital. Case 26-2011. A 7-year-old boy with a complex cyst in the kidney. N Engl J Med 2011;365:743-51.

8. Back SJ, Andronikou S, Kilborn T, Kaplan BS, Darge K. Imaging features of tuberous sclerosis complex with autosomal-dominant polycystic kidney disease: a contiguous gene syndrome. Pediatr Radiol 2015;45:386-95.
9. Cabrera-López C, Bullich G, Martí T, Català V, Ballarín J, Bissler JJ, et al. Insight into response to mTOR inhibition when PKD1 and TSC2 are mutated. BMC Med Genet 2015;16:39.

10. Longa L, Scolari F, Brusco A, Carbonara C, Polidoro S, Valzorio B, et al. A large TSC2 and PKD1 gene deletion is associated with renal and extrarenal signs of autosomal dominant polycystic kidney disease. Nephrol Dial Transplant 1997;12:1900-7.

11. Oyazato $Y$, lijima K, Emi M, Sekine T, Kamei K, Takanashi J, et al. Molecular analysis of TSC2/PKD1 contiguous gene deletion syndrome. Kobe J Med Sci 2011;57:E1-10.

12. Llamas Velasco S, Camacho Salas A, Vidales Moreno C, Ceballos Rodríguez RM, Murcia García FJ, Simón de la Heras R. TSC2/PKD1 contiguous gene deletion syndrome. An Pediatr (Barc) 2013;79:42-5.

13. Kacerovska D, Vrtel $R$, Michal M, Vanecek $T$, Vodicka R, Kreuzberg $B$, et al. TSC2/PKD1 contiguous gene syndrome: a report of 2 cases with emphasis on dermatopathologic findings. Am J Dermatopathol 2009;31:532-41.

14. Ismail NF, Nik Abdul Malik NM, Mohseni J, Rani AM, Hayati F, Salmi $A R$, et al. Two novel gross deletions of TSC2 in Malaysian patients with tuberous sclerosis complex and TSC2/PKD1 contiguous deletion syndrome. Jpn J Clin Oncol 2014;44:506-11.

15. Sampson JR, Maheshwar MM, Aspinwall R, Thompson P, Cheadle JP,

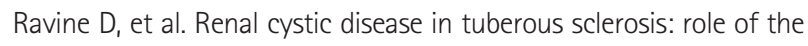
polycystic kidney disease 1 gene. Am J Hum Genet 1997;61:843-51.

16. Hajj P, Ferlicot S, Massoud W, Awad A, Hammoudi Y, Charpentier B, et al. Prevalence of renal cell carcinoma in patients with autosomal dominant polycystic kidney disease and chronic renal failure. Urology 2009;74:631-4.

17. Bonnet CS, Aldred M, von Ruhland $C$, Harris $R$, Sandford $R$, Cheadle JP. Defects in cell polarity underlie TSC and ADPKD-associated cystogenesis. Hum Mol Genet 2009;18:2166-76.

18. Walz G, Budde K, Mannaa M, Nürnberger J, Wanner C, Sommerer $\mathrm{C}$, et al. Everolimus in patients with autosomal dominant polycystic kidney disease. N Engl J Med 2010;363:830-40.

19. Bisceglia M, Galliani C, Carosi I, Simeone A, Ben-Dor D. Tuberous sclerosis complex with polycystic kidney disease of the adult type: the TSC2/ADPKD1 contiguous gene syndrome. Int J Surg Pathol 2008;16:375-85. 